\title{
Controlling Diffusion by Varying Width of Layers in Nano Channel
}

\author{
Ishu Goyal ${ }^{1}$, Sunita Srivastava ${ }^{1}$, K. Tankeshwar ${ }^{2 *}$
}

(Received 25 June 2012; accepted 20 August 2012; published online 25 September 2012.)

\begin{abstract}
Diffusive dynamics of fluid forming layers of high and low density regions in a nanochannel has been investigated. Diffusion coefficient in direction parallel and perpendicular to the confining wall has been found to show behaviour which is not observed in micro channel or bulk systems. The behaviour of diffusion is found to be controlled by the width of layers formed in nanochannel due to wall and particle interactions. This is an important result as width of layers and hence flow of fluid inside nano pores/tube can be controlled by an external source.
\end{abstract}

Keywords: Nanochannel; Layer; Diffusion

Citation: Ishu Goyal, Sunita Srivastava and K. Tankeshwar, "Controlling Diffusion by Varying Width of Layers in Nano Channel", Nano-Micro Lett. 4 (3), 154-157 (2012). http://dx.doi.org/10.3786/nml.v4i3. p154-157

\section{Introduction}

Controlling behavior of matter on nanoscale is of great interest as it allows us to exploit and control the nanoscale devices, which has wide applications in chemistry, polymer industry, tribology and lubrication, as well as in fabrication of MEMS (micro-electromechanical-system) and biochemical lab-on-chip systems. The study of confined fluids in nano scale is quite different from the fluid confined in micro or larger length scale. One of the important transport properties of fluid, for example, diffusion at micro/nano scale confinement has already been addressed by many researchers. Anisotropic diffusion [1] varies with distance from confining walls and it shows sharp decrease near the confining wall. Different widths of channel/pore diffusion show different behavior. Karniadakis et al. [2] observed that mobility of fluid is maintained even in pore widths of $h=2.0 \sigma$ ( $h$ is pore width and $\sigma$ is length parameter), while diffusion parallel to the pore wall below $h=4.0 \sigma$ becomes an oscillating function of the channel width and beyond $4.0 \sigma$. The diffusivity in- creases smoothly and reaches the bulk value of $11.57 \sigma$. Wei et al. [3] studied the diffusion of water in slits of rutile $\mathrm{TiO}_{2}$ (110) and graphite (0001) ranging from 0.8 $\mathrm{nm}$ to $2 \mathrm{~nm}$ by using Molecular Dynamics (MD) simulation and observed that self diffusion of the bound water is almost zero on the surface of $\mathrm{TiO}_{2}$. Sofos et al. [4] studied the planar Poiseuille nanoflow between liquid argon and krypton walls. The channel width $(h)$ is found to be in the range of $2.65 \sigma-18.58 \sigma$. The calculated diffusion coefficients in distinct layers parallel to walls reveal that diffusion is higher in layers which is close to the center of the channel and decreases for the layers adjacent to the walls. The behavior of confined fluids in nanoslit pores has also been studied by Tanmineh et al. [6]. In all cases it is concluded that the global (i.e., averaged over the nanochannel volume) dynamics of the confined liquids are slower than those in the bulk. Recent work on water in nano confinement focuses on structures with diameters in the 1-2 $\mathrm{nm}$ range. And carbon nanotubes were used as the main theoretical and experimental model because of the similarities in terms of dimensions and hydrophobicity to pores in

\footnotetext{
${ }^{1}$ Department of Physics, Panjab University, Chandigarh-160014, India

${ }^{2}$ Computer Centre, Panjab University, Chandigarh-160014, India

*Corresponding author. E-mail: tankesh@pu.ac.in
} 
biological systems.

Depending on the nature of the strength of the wallfluid interactions, the confined fluid may undergo phase transition as the confining walls are moved closer, which is different from the bulk fluid. These changes belong to the arena of excluded volume effects and are significant when the confining dimensions are less than five to ten times the diameter of fluid constituents. In this limit, a structural force acts on the confining surfaces that oscillate. According to contact value theorem, a positive (negative) force reflects an enhanced (lowered) local density near the confining walls. Thus, a layered (disordered) density is present next to the walls. Intuitively, the density oscillation induced by each of the two walls interfere constructively or destructively, depending on whether an integer or half integer number of layers fit into the confining channels $[7,8]$. These density oscillations at the interface arise from short distance interactions between surfaces which are known as solvation forces or structural forces. The period of these oscillations roughly equals the dimensions of a liquid molecule. When walls are well separated, the layering breaks down as density fluctuations induced by the walls no longer overlap sufficiently. So, the separation induced by well-ordered layering wall should be small.

New dynamical behavior of confined fluids include: the density oscillations near the solid wall, the enhanced effects on shear viscosity, the prolonged relaxation time, the transition to solidification, reduced diffusion coefficient etc. Constrained molecular motion in confined fluid dramatically increases the relaxation time as compared to bulk fluids and thus the solid like behavior emerges. However, such conventional behavior is not always true. For example, conventionally, in bulk fluid with higher density has lesser value of self diffusion coefficient. However, for fluid confined to nano-dimensions, self-diffusion coefficient normal to confined walls has shown [9] reversal in its behavior. The fluid has more diffusion coefficient in region of high density. The reason to this reversal has been attributed to mechanism of formation of layers for hard spheres. The study of Mittal et al. [9] restricts to fixed width of layers of alternatively high density region and low density regions, thereby, implying that density profile along direction normal to wall had fixed frequency. Thus, the space provided to diffuse a particle is affected by varied packing fraction.

\section{Theoretical method}

In the present work, we consider fluid enclosed by two smooth walls such that it has nanodimensions in one of the directions (say $z$ direction). The fluid considered here is Lennard Jones fluid near the triple point (reduced density $n \sigma^{3}=0.844$ and reduced temperature $k T / \varepsilon=0.73)$ and is confined to a nanochannel of width of five times of the atomic diameter i.e. less than $2 \mathrm{~nm}$. Here, fluid forms layers of alternatively low and high density regions. The width of layers can be varied by controlling strength of wall interactions with fluid particles. Spatially varying density profile can be defined as

$$
\rho(z)=\frac{\cos (\Omega z)}{a}+\rho .
$$

In above equation, $\rho$ is the density of fluid in bulk (in the absence of wall/pores etc.), ' $a$ ' is the adjustable parameter determining the deviation from bulk value. $\Omega$ is a parameter; for different values of $\Omega$ we can have different density profile with layers of different width. We start examining the behavior of restoring frequency (second sum rule of velocity auto correlation function). The expressions for restoring frequency are different for parallel and perpendicular directions $[10,11]$. These are respectively given by

$$
\begin{aligned}
V_{2}^{\|}\left(z_{1}\right)= & \int_{-\infty}^{\infty} d x_{12} \int_{-\infty}^{\infty} d y_{12} \int_{-l}^{l} d z_{2} \rho\left(z_{2}\right) g\left(R_{12}\right) \times \\
& {\left[\frac{x_{12}^{2}}{R_{12}^{2}}\left(\frac{\partial^{2} U\left(R_{12}\right)}{\partial R_{12}^{2}}-\frac{\partial U\left(R_{12}\right)}{\partial R_{12}} \frac{1}{R_{12}}\right)+\right.} \\
& \left.\frac{\partial U\left(R_{12}\right)}{\partial R_{12}} \frac{1}{R_{12}}\right], \\
V_{2}^{\perp}\left(z_{1}\right)= & \int_{-\infty}^{\infty} d x_{12} \int_{-\infty}^{\infty} d y_{12} \int_{-l}^{l} d z_{2} \rho\left(z_{2}\right) g\left(R_{12}\right) \times \\
& {\left[\frac{z_{12}^{2}}{R_{12}^{2}}\left(\frac{\partial^{2} U\left(R_{12}\right)}{\partial R_{12}^{2}}-\frac{\partial U\left(R_{12}\right)}{\partial R_{12}} \frac{1}{R_{12}}\right)+\right.} \\
& \left.\frac{\partial U\left(R_{12}\right)}{\partial R_{12}} \frac{1}{R_{12}}\right] .
\end{aligned}
$$

where

$$
R_{12}^{2}=x_{12}^{2}+y_{12}^{2}+z_{12}^{2},
$$

and $z_{12}=z_{1}-z_{2}$ with $z_{1}$ and $z_{2}$ as the co-ordinates in $z$ direction for pair of particles. $2 l$ is the width of channel in $\mathrm{z}$ direction varying from $-l$ to 1 . In the present case we take $l=2.5 \sigma, U(r)$ is the Lennard Jones potential, $g(R)$ is the radial distribution function taken from the work of Sung \& Chandler [12], $\rho(z)$ is the spatially varying density of fluid which varies in z direction only is dependent upon ' $a$ ' and $\Omega$.

The pair contribution to fourth frequency sum rules for parallel and perpendicular cases are given as

$$
V_{42}^{\| l}\left(z_{1}\right)=2 \int_{-\infty}^{\infty} d x_{12} \int_{-\infty}^{\infty} d y_{12} \int_{-l}^{l} d z_{2} \rho\left(z_{2}\right) g\left(R_{12}\right) \times
$$




$$
\begin{aligned}
& {\left[\frac{x_{12}^{2}}{R_{12}^{2}}\left(\frac{\partial^{2} U\left(R_{12}\right)}{\partial R_{12}^{2}}-\frac{\partial U\left(R_{12}\right)}{\partial R_{12}} \frac{1}{R_{12}}\right)+\right.} \\
& \left.\frac{\partial U\left(R_{12}\right)}{\partial R_{12}} \frac{1}{R_{12}}\right]^{2},
\end{aligned}
$$

and

$$
\begin{aligned}
V_{42}^{\perp}\left(z_{1}\right)= & 2 \int_{-\infty}^{\infty} d x_{12} \int_{-\infty}^{\infty} d y_{12} \int_{-l}^{l} d z_{2} \rho\left(z_{2}\right) g\left(R_{12}\right) \times \\
& {\left[\frac{z_{12}^{2}}{R_{12}^{2}}\left(\frac{\partial^{2} U\left(R_{12}\right)}{\partial R_{12}^{2}}-\frac{\partial U\left(R_{12}\right)}{\partial R_{12}} \frac{1}{R_{12}}\right)+\right.} \\
& \left.\frac{\partial U\left(R_{12}\right)}{\partial R_{12}} \frac{1}{R_{12}}\right]^{2} .
\end{aligned}
$$

Dynamical model $[10,11]$ to study self-diffusion for the fluid confined in nanoscale has revealed that when fluid is confined to dimensions about twenty times of atomic diameter, a considerable decrease in diffusion occurs when one moves towards the walls from the centre of the nanochannel. The density of fluid throughout was assumed to be uniform. Restricting to simple and reliable model [13] for the calculation of diffusion coefficient as judged from comparison of its results with simulation data for various systems [14-16], the expression for the self-diffusion coefficient $D$ is given by:

$$
D=\frac{k_{B} T}{m} \frac{\pi}{2} \tau \sec h\left(\frac{\pi \omega \tau}{2}\right) .
$$

where, $\tau$ and $\omega$ in the above equation are given as

$$
\tau^{-2}=\delta_{2} / 4, \quad \omega^{2}=\left(4 \delta_{1}-\delta_{2}\right) / 4 .
$$

Here, $\delta_{1}=V_{2}$ and $\delta_{2}=\left(V_{4} / V_{2}-V_{2}\right)$.

\section{Results and discussion}

Diffusion in direction normal and parallel to wall is obtained from Eq. (5) by using corresponding values of $V 2$ and $V 4$ obtained from expressions (2) and (4). The results obtained are presented below as a function of $z$. $z=0$ represents the center of the confined geometry. These results are shown for different values of $\Omega$ which determine the layer widths of higher and lower density regions as shown in the Fig. 1 for density profile $\rho(z)$. From the Fig. 1, it can be seen that diffusion coefficients parallel and normal to confined walls show oscillations. It is interesting to see that at the centre of geometry normal and parallel diffusions both show reversal (maxima to minima or from minima to maxima) in the behavior on varying $\Omega$ from 3 to 6.5 in spite of the fact that density at $z=0$ remains maximum. This is in contradiction to conventional dependence of diffusion on density. At $\Omega=3$ parallel diffusion shows minimum and normal diffusion shows maximum. At this value of
$\Omega=3$ the width of high density region is more than one atomic diameter and diffusing particle is able to find sufficient space to move in perpendicular direction. On increasing $\Omega$ layers of high density regions and low density regions becomes narrow. More fractions of particles puts away their energy by moving only in the $x-y$ plane and hence parallel diffusion increases and perpendicular diffusion decreases. At $\Omega=6.5$, normal diffusion at $z=0$ becomes minimum, whereas parallel diffusion has maximum. These results are very interesting and show how dynamics of fluid changes with change in size and layer width. The flow of fluid at given density in a nanotube/nano pore can be controlled by width of layers. Width of layers, in turn, can be controlled by external forces or its interaction of walls with particles. This study can have many applications in nanofluidics and biological systems where control over the fluid flow is required.

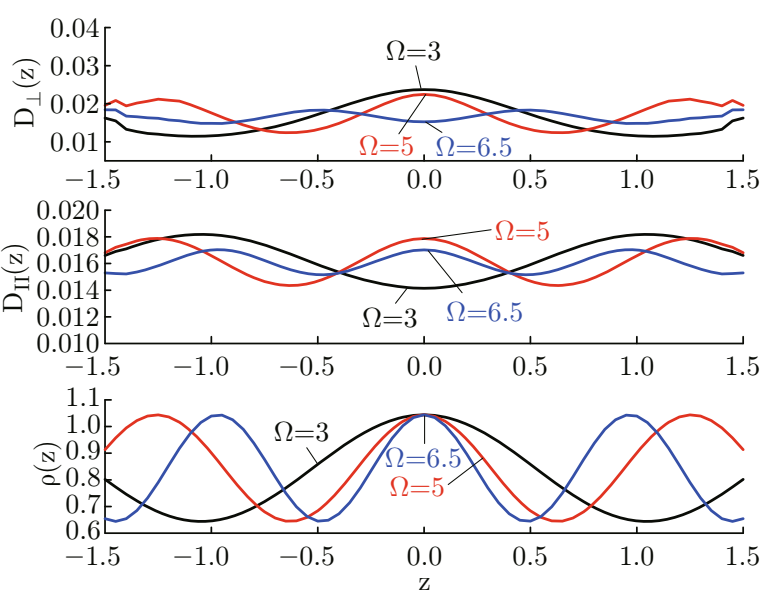

Fig. 1 Variation of parallel $(D / /)$ and perpendicular $(D \perp)$ diffusion coefficients versus $z$ for $\Omega=3,5,6.5$. Density profile $\rho(z)$ vs $z$ is also shown.

The study can be examined further by examining normal and parallel by looking the behavior of short time properties of correlation of normal and parallel velocity auto correlation function. It is seen that parallel and perpendicular parts of $V 2$ show variations which are opposite to each other. Maxima of parallel $V 2$ coinciding with minimum of normal part. Diffusion which varies inversely of frequency $\sqrt{ } \mathrm{V} 2$ found to follow more or less same trend which implies that short time dynamics of the confined liquids is also quite different from the bulk fluids.

\section{Conclusion}

In conclusion, theoretical results for diffusion of fluid confined to a nanochannel of width 5 times of the atomic diameter have been demonstrated. It has been found in our study that confinement of the fluid to such 
a length scale provides anomalous but interesting direction dependence to diffusion coefficient. The width of layers formed is found to control the behaviour of diffusion.

\section{References}

[1] N. Aggarwal, J. Sood and K. Tankeshwar, Nanotechnology 18, 335707 (2007). http://iopscience.iop. org/0957-4484/18/33/335707

[2] G. E. Karniadakis, A. Beskok and N. Aluru, Microflows: Fundamentals and Simulation, Springer, 2002.

[3] M. J. Wei, J. Zhou, X. Lu, Y. Zhu, W. Liu, L. Lu and L. Zhang, Fluid Phase Equilib. 302, 316 (2011). http://www.sciencedirect.com/ science/article/pii/S0378381210005121

[4] F. Sofos, T. Karakasidis and A. Liakopoulos, Int. J. Heat Mass Tran. 52, 735 (2009). http:// www.sciencedirect.com/science/article/pii/ S0017931008004407

[5] F. Sofos, T. E. Karakasidis and A. Liakopoulos, Int. J. Heat Mass Tran. 53, 3839 (2010). http:// www.sciencedirect.com/science/article/pii/ S0017931010002346

[6] T. Keshavarzi, F. Sedaghat and G. A. Mansoori, Micro?uid Nano?uid 8, 97 (2010). http://www . springerlink. com/content/vr14247607036366/

[7] O. Bunk, et al., Phys. Rev. E 75, 021501 (2007). http://pre.aps.org/abstract/PRE/v75/i2/e021501
[8] C. J. Hemming and G. N. Patey, J. Chem. Phys. 121, 6508 (2004). http://jcp.aip.org/resource/1/ jcpsa6/v121/i13/p6508_s1?isAuthorized=no

[9] Jeetain Mittal, Thomas M. Truskett, Jeffrey R. Errington and Gerhard Hummer, Phys. Rev. Lett 100, 145901 (2008). http://prl.aps.org/abstract/PRL/ v100/i14/e145901

[10] K. Tankeshwar and S. Srivastava, Nanotechnology 18, 485714 (2007). http://iopscience.iop.org/ 0957-4484/18/48/485714

[11] R. Devi, J. Sood, S. Srivastava and K. Tankeshwar, Microfluid Nanofluid 9, 737 (2010). http://www . springerlink. com/content/d117n48674371585/

[12] S. Sung and D. Chandler, J. Chem. Phys. 56, 4986 (1972). http://gold.cchem. berkeley.edu/Pubs/ DC12.pdf

[13] K. Tankeshwar, B. Singla and K. N. Pathak, J. Phys. Condens. Matter 3, 3173 (1991). http://iopscience. iop.org/0953-8984/3/18/011

[14] K. Tankeshwar and M. P. Tosi, J. Phys.: Condensed Matter 3, 7511(1991). http://iopscience.iop.org/ 0953-8984/3/38/022

[15] K. Tankeshwar and M. P. Tosi, J. Phys.: Condens. Matter 3, 9817 (1991). http://iopscience.iop.org/ 0953-8984/3/48/023

[16] R. K. Sharma, K. Tankeshwar and K. N. Pathak, J. Phys.: Condens. Matter 7, 537 (1995). http:// iopscience.iop.org/0953-8984/7/3/009 